\title{
THE BONNET PROBLEM FOR HARMONIC MAPS TO THE 3-SPHERE
}

\author{
BART DIOOS AND JOERI VAN DER VEKEN
}

\begin{abstract}
In previous work [10] the authors defined transforms for nonconformal harmonic maps from a Riemann surface into the 3-sphere. An observation from that study was that two invariants, a real and a complex function, determine a non-conformal harmonic map up to isometries of the 3-sphere. We show that if the first invariant of a harmonic map and its transformed map are the same, then these maps are either congruent or the harmonic map belongs to a particular 1-parameter family. Inspired by this result we discuss the Bonnet problem for non-conformal harmonic maps: to what extent is a harmonic map determined by its first invariant?
\end{abstract}

\section{IntRODUCTION}

A map $f: S \rightarrow S^{3}$ from a Riemann surface $S$ into the 3 -sphere is harmonic if it satisfies the equation $\Delta f+|d f|^{2} f=0$ where $\Delta$ is the Laplacian on the surface $S$. The map is conformal if it preserves the conformal structure on $S$. In this case the map is a minimal immersion of the surface in the 3 -sphere. In this article however we will discuss non-conformal harmonic maps.

In a previous paper [10] of the authors it was shown that from a non-conformal harmonic map $f$ from a surface to $S^{3}$ one can construct two new maps of the same type, denoted by $f^{+}$and $f^{-}$. These two constructions, called the $(+)$and $(-)$ transforms, are mutually inverse in the sense that applying successively and in any order the $(+)$ and the $(-)$ construction gives the original map. The transforms are inspired by the works [1] and [4] on minimal surfaces in odd-dimensional spheres and are natural generalisations of the polar surface of minimal surfaces in $S^{3}$ ([13]). Taking twice the polar surface of a minimal surface in $S^{3}$ produces the original surface. This is however not the case for the transforms for non-conformal harmonic maps. By repeatedly applying one of the transforms on an initial non-conformal harmonic map one obtains a sequence of such maps.

It is possible that all harmonic maps in the sequence are equal to each other up to isometries of $S^{3}$. Non-conformal harmonic maps are characterized up to an isometry of $S^{3}$ by two invariants, a real-valued function $\phi$ and a complex-valued function $\mu$, satisfying two integrability conditions (see Proposition 2.1 for a correct formulation). Thus a non-conformal harmonic map is congruent to its transformed harmonic map if and only if the invariants of the harmonic map and the transformed map agree, that is, if $\phi=\phi^{\varepsilon}$ and $\mu=\mu^{\varepsilon}$ (here $\varepsilon$ stands for + or - ). In this case all the maps in the associated sequence are congruent and are certain reparametrisations of Clifford tori. Moreover both invariants are then constant (Theorem 3.3).

2010 Mathematics Subject Classification. Primary 53C43; Secondary 58E20, 53C42.

Key words and phrases. 3-sphere, Bonnet problem, harmonic maps.

This work is partially supported by the Belgian Interuniversity Attraction Pole P07/18 (Dygest) and project 3E160361 (Lagrangian and calibrated submanifolds) of the KU Leuven Research Fund. 
In fact we proved a slightly better result. It is sufficient that $\mu=\mu^{\varepsilon} \neq 0$ such that the harmonic map and its transform are congruent. In view of this result, it is natural to ask what happens when the first invariants $\phi$ and $\phi^{\varepsilon}$ agree. It turns out that this is not the case: there exists essentially one harmonic map $f$ whose first invariant $\phi$ agrees with the first invariant $\phi^{\varepsilon}$ of its transformed map $f^{\varepsilon}$ such that $f$ and $f^{\varepsilon}$ are not congruent.

Theorem 1.1. Consider a non-conformal harmonic map $f: S \rightarrow S^{3}$ from a Riemann surface $S$ to the three-sphere. Let $f^{\varepsilon}$ be its $\varepsilon$-transform $(\varepsilon=+$ or $\varepsilon=-)$ and assume that $\phi=\phi^{\varepsilon}$. Then either $f$ is congruent to $f^{\varepsilon}$ or, after a suitable choice of complex coordinate on $S$, the invariants of $f$ are

$$
\phi=\operatorname{arcsinh}\left(\frac{1}{\sqrt{|z|}}\right), \quad \quad \mu=-\frac{2 \varepsilon}{z} \frac{C(|z|+1)+i}{C \sqrt{|z|+1}}
$$

for some real constant $C>0$.

The proof of Theorem 1.1 hinges on the fact that the level curves of an isoparametric function from the plane are parallel lines or concentric circles (see [2] or [14]).

Inspired by this theorem, we arrive at the following problem for non-conformal harmonic maps. Consider two non-conformal harmonic maps whose first invariants are equal to each other. Are the two harmonic maps then necessarily congruent? This problem will be called the Bonnet problem for non-conformal harmonic maps. It is clear that this problem is a variation of the classical Bonnet problem for CMC surfaces in Euclidean three-space [5]. The Bonnet problem has also been investigated for surfaces in $S^{3}$ and $H^{3}$ (see [3], [6], [7], [8], [9]). More recently the problem was extended to surfaces in homogeneous three-spaces with a four-dimensional isometry group [11] and Lagrangian surfaces in complex space forms $[12]$.

Eventhough this problem is similar to the classical Bonnet problem for constant mean curvature (CMC) surfaces, there is an essential difference. Every CMC surface in Euclidean three-space admits a 1-parameter family of CMC surfaces. If $Q d z^{2}$ is the Hopf differential of a surface with CMC $H$, then for every real number $t$ the map $e^{i t} Q$ satisfies the Gauss-Codazzi equations for surfaces with CMC $H$, thus giving a CMC surface for every value of the parameter $t$. The metric remains invariant under this transformation of the Hopf differential, so the 1-parameter family of CMC surfaces is obtained by isometric deformations. Non-conformal harmonic maps also admit a 1-parameter family of harmonic maps, however, the first invariant $\phi$ does not remain invariant under the deformation for non-conformal harmonic maps (see Lemma 5.1).

The outline of the paper is as follows. The first two sections summarise the necessary background from [10]. In Section 2 we introduce an adapted complex coordinate and state the existence and uniqueness theorem for non-conformal harmonic maps into $S^{3}$ In Section 3 we recall the definition of two transforms for harmonic maps and the construction of a sequence of harmonic maps using these two transforms. The main theorem Theorem 1.1 is proven in Section 4 . In the last section we formulate the Bonnet problem for non-conformal harmonic maps and point out the similarities and differences with the classical Bonnet problem for CMC surfaces.

The authors would like to thank Makoto Sakaki for his helpful comments and remarks.

\section{HARMONIC MAPS TO $S^{3}$}

It is convenient to use quaternions to describe harmonic maps from a surface into the 3 -sphere. The ring of quaternions $\mathbb{H}$ can be identified with the vector space $\mathbb{R}^{4}$. 
Writing quaternions as real linear combinations of the basis elements $1, e_{1}, e_{2}$ and $e_{3}$, quaternion multiplication is determined completely by distibutivity and the identities

$$
e_{1}^{2}=e_{2}^{2}=e_{3}^{2}=e_{1} e_{2} e_{3}=-1
$$

A quaternion that is a linear combination of $e_{1}, e_{2}$ and $e_{3}$ only is called an imaginary quaternion. The set of imaginary quaternions $\operatorname{Im} \mathbb{H}$ can be identified with the Euclidean space $\mathbb{R}^{3}$. The product of two imaginary quaternions $\alpha$ and $\beta$ is given by

$$
\alpha \beta=-\langle\alpha, \beta\rangle+\alpha \times \beta
$$

where $\langle$,$\rangle is the Euclidean inner product and \times$ is the vector product on $\mathbb{R}^{3}$.

Let us now describe the 3 -sphere and its tangent spaces. The 3 -sphere $S^{3}$ is the set of unit quaternions $\{p \in \mathbb{H} \mid\langle p, p\rangle=1\}$. One can prove that $\langle u v, u w\rangle=$ $\langle u, u\rangle\langle v, w\rangle$ for all quaternions $u, v$ and $w$. Therefore $p \alpha$ is orthogonal to $p$ for every imaginary quaternion $\alpha$ and the tangent space at $p$ is

$$
T_{p} S^{3}=\{p \alpha \mid \alpha \in \operatorname{Im} \mathbb{H}\} .
$$

On a surface we will use complex coordinates, so in order to describe the complexified tangent vectors we will need the complexified quaternions $\mathbb{H} \otimes \mathbb{C}=\mathbb{H} \oplus i \mathbb{H}$. The element $i$ must be distinguished from the imaginary units $e_{i}$ in $\mathbb{H}$. The complex bilinear extension of the Euclidean metric and vector product will also be denoted by $\langle$,$\rangle and \times$. The product of two complexified quaternions $p_{1}+i p_{2}$ and $q_{1}+i q_{2}$ is

$$
\left(p_{1}+i p_{2}\right)\left(q_{1}+i q_{2}\right)=\left(p_{1} q_{1}-p_{2} q_{2}\right)+i\left(p_{1} q_{2}+p_{2} q_{1}\right)
$$

Now we consider a harmonic map $f: S \rightarrow S^{3} \subset \mathbb{H}$ from a Riemann surface $S$ into the 3 -sphere $S^{3}$. Choose a local complex coordinate $z=x+i y$ on $S$. Derivations with respect to a complex coordinate $z$ or the real coordinates $x$ and $y$ will be denoted by $f_{z}, f_{x}$ and $f_{y}$ respectively. Since $\langle f, f\rangle=1$, it follows that $\left\langle f, f_{z}\right\rangle=0$.

Harmonicity of the map means that $f_{z \bar{z}}=-\left|f_{z}\right|^{2} f$. If $f$ is in addition a conformal map, $f$ is a minimal isometric immersion of $S$ in $S^{3}$. In this paper we assume that $f$ is not conformal. By the harmonicity of $f$, the quadratic differential $\Theta=$ $\left\langle f_{z}, f_{z}\right\rangle d z^{2}$ is holomorphic. Since $f$ is assumed to be non-conformal, $\left\langle f_{z}, f_{z}\right\rangle$ is non-zero. Therefore there exists a complex coordinate $z$ such that $\left\langle f_{z}, f_{z}\right\rangle=-1$. Geometrically this means that the coordinate lines of the image surface $f(S)$ in the three-sphere are orthogonal. We will call such a coordinate an adapted complex coordinate for $f$.

By (3) there exist functions $\alpha$ and $\beta$ with values in $\operatorname{Im} \mathbb{H}$ such that $f_{x}=f \alpha$ and $f_{y}=f \beta$. This means that $f_{z}=\frac{1}{2} f(\alpha-i \beta)$. It follows from $\left\langle f_{z}, f_{z}\right\rangle=-1$ that

$$
\langle\alpha, \alpha\rangle-\langle\beta, \beta\rangle=-4, \quad\langle\alpha, \beta\rangle=0 .
$$

Hence there is a non-negative smooth function $\phi$ such that

$$
|\alpha|=2 \sinh \phi, \quad|\beta|=2 \cosh \phi .
$$

Note that $\beta$ is nowhere vanishing by equation (4), but $\alpha$ can be zero. At points were $\alpha$ is not zero the vectors $f_{z}$ and $f_{\bar{z}}$ are linearly independent and $\phi$ is positive. In the following we will assume that $f$ is an immersion. Since our discussions are always local, we may restrict ourselves to the subset $U$ of $S$ where $\alpha \neq 0$. On this set $\phi$ is positive and the image $f(U)$ is a surface in the 3 -sphere. At a point of $U$ we define $N$ to be the real unit vector in the positive direction of $f(\alpha \times \beta)$. Then the normal vector $N$ is given by

$$
N=\frac{1}{2} \operatorname{csch} 2 \phi f(\alpha \times \beta) .
$$


We now have defined a complex moving frame $\mathcal{F}=\left\{f, f_{z}, f_{\bar{z}}, N\right\}$ for the harmonic map $f$ on the set $U$. The moving frame equations for $\mathcal{F}$ are

$$
\begin{aligned}
f_{z z} & =f+2 \phi_{z}\left(\operatorname{coth} 2 \phi f_{z}+\operatorname{csch} 2 \phi f_{\bar{z}}\right)+\mu N, \\
f_{\bar{z} z} & =-\cosh 2 \phi f, \\
N_{z} & =-\mu \operatorname{csch} 2 \phi\left(\operatorname{csch} 2 \phi f_{z}+\operatorname{coth} 2 \phi f_{\bar{z}}\right),
\end{aligned}
$$

where $\mu=\left\langle f_{z z}, N\right\rangle$. The derivatives with respect to $\bar{z}$ can be found by complex conjugating the above equations. The integrability conditions $\mathcal{F}_{z \bar{z}}=\mathcal{F}_{\bar{z} z}$ for the frame $\mathcal{F}$ give

$$
\begin{aligned}
2 \phi_{z \bar{z}} & =-\sinh 2 \phi+|\mu|^{2} \operatorname{csch} 2 \phi, \\
\mu_{\bar{z}} & =-2 \bar{\mu} \phi_{z} \operatorname{csch} 2 \phi .
\end{aligned}
$$

The real function $\phi$ is a measure for the non-conformality of $f$ at a point. The complex function $\mu$ measures the rate at which the image of $f$ is pulling away from the great 2 -sphere tangent to the image of $f$. If $f$ is a map into a great 2 -sphere, then $\mu$ vanishes and the first integrability condition reduces to the sinhGordon equation.

We end this section with a Bonnet-type existence and uniqueness result for nonconformal harmonic maps into $S^{3}$. A non-conformal harmonic map from a Riemann surface to $S^{3}$ are completely characterized by the invariants $\phi$ and $\mu$. The following proposition follows directly from Proposition 4.1 in [10].

Proposition 2.1. Let $f_{1}, f_{2}: S \rightarrow S^{3}$ be two non-conformal harmonic maps with the same adapted coordinate $z$. Let $\phi_{1}, \mu_{1}$ and $\phi_{2}, \mu_{2}$ be the invariants of $f_{1}$ respectively $f_{2}$. If $\phi_{1}=\phi_{2}$ and $\mu_{1}=\mu_{2}$, then there is orientation preserving isometry $F$ such that $f_{1}=F \circ f_{2}$. If $\phi_{1}=\phi_{2}$ and $\mu_{1}=-\mu_{2}$, then there is orientation reversing isometry $F$ such that $f_{1}=F \circ f_{2}$.

\section{TRANSFORMS OF HARMONIC MAPS TO $S^{3}$}

Let $f: S \rightarrow S^{3}$ be a non-conformal harmonic map with an adapted coordinate $z$. In this section we will explain how to associate to $f$ two other non-conformal harmonic maps $f^{+}$and $f^{-}$from $S$ to $S^{3}$. With these transforms one can construct a sequence $\left\{f^{p} \mid p \in \mathbb{Z}\right\}$ of non-conformal harmonic maps from $S$ to $S^{3}$ starting with the map $f^{0}=f$.

Fix a point $p \in S$ and consider the vectors

$$
\pm \sin \theta \frac{f \beta}{|f \beta|}+\cos \theta N
$$

in $T_{f(p)} S^{3}$, where $\theta$ is chosen such that $\cos \theta=|\alpha| /|\beta|=\tanh \phi$ and $\sin \theta=\operatorname{sech} \phi$. The ellipse $E$ with $f \alpha$ and $f \beta$ as minor and major semi-axes is the image of a circle in the tangent plane to $S$ at $p$ under $d f$. The $\operatorname{cosine} \cos \theta$ is the ratio between the lengths of the minor and major axes of this ellipse and is a measure for its eccentricity. The vectors (7) have a nice geometric meaning. Let $R_{\theta}$ be the rotation of $T_{f(p)} S^{3}$ about the minor axis of $E$ through the angle $\theta$. Then the orthogonal projection of the rotated ellipse $R_{\theta}(E)$ onto the plane containing $E$ is a circle. The same holds for the rotation $R_{-\theta}=R_{\theta}^{-1}$. The vectors above are the images of the unit normal $N$ under the rotations $R_{\theta}$ and $R_{-\theta}$.

We can rewrite the vectors in (7) as

$$
f^{ \pm}=\frac{1}{2} \operatorname{sech}^{2} \phi\left( \pm \beta+\frac{1}{2} \alpha \times \beta\right) .
$$

By varying the point $p$, we can regard $f^{+}$and $f^{-}$as maps from $S$ to $S^{3}$ again and we call them the $(+)$ transform and $(-)$ transform of $f$ respectively. If $f$ were 
conformal, that is if $|\alpha|=|\beta|$ everywhere, the expressions (7) still make sense. In fact, we get $\theta=0$ and recover the polar surface of the minimal surface $f$ (see [13]).

From now on we restrict to the non-conformal case and we denote the transforms (8) by $f^{\varepsilon}$ where $\varepsilon$ is 1 or -1 . All objects and functions related to $f^{\varepsilon}$ will be denoted with a superscript $\varepsilon$. For instance, the function $\phi^{\varepsilon}$ is the non-negative smooth function that satisfies the $\varepsilon$-analogue of (4).

Theorem 3.1 ([10]). Let $f: S \rightarrow S^{3}$ be a non-conformal harmonic map from a Riemann surface $S$ into the 3-sphere and $z$ an adapted coordinate for $f$. Then the tranforms $f^{\varepsilon}, \varepsilon= \pm 1$, are also non-conformal harmonic maps from $S$ to $S^{3}$ and $z$ is also an adapted coordinate for $f^{\varepsilon}$. Furthermore the $(+)$ transform and $(-)$ transform of $f$ are mutual inverses in the sense that

$$
\left(f^{+}\right)^{-}=\left(f^{-}\right)^{+}=f .
$$

It can be shown that the function $\phi^{\varepsilon}$ is positive on an open dense subset of $S$. Therefore the moving frame $\mathcal{F}^{\varepsilon}=\left\{f^{\varepsilon}, f_{1}^{\varepsilon}, \bar{f}_{1}^{\varepsilon}, N^{\varepsilon}\right\}$ of a transformed harmonic map $f^{\varepsilon}$ is well-defined on this subset. The $\varepsilon$-versions of the moving frame equations and integrability conditions for $\mathcal{F}^{\varepsilon}$ hold as well on this subset. Since our calculations are local, we may tacitly assume that we are working on an open set where the invariants $\phi$ and $\phi^{\varepsilon}$ are positive.

Theorem 3.1 allows us to associate to a non-conformal harmonic map $f: S \rightarrow S^{3}$ a sequence $\left\{f^{p} \mid p \in \mathbb{Z}\right\}$ of such harmonic maps by defining $f^{0}=f, f^{p+1}=\left(f^{p}\right)^{+}$ and $f^{p-1}=\left(f^{p}\right)^{-}$for every integer $p$. Moreover, if $z$ is an adapted complex coordinate for one of the maps $f^{p}$ in the sequence, then it is an adapted complex coordinate for every map in the sequence. This sequence is called the sequence associated to the map $f$.

The invariants of $f$ and those of the transformed map $f^{\varepsilon}$ are related by the following relations.

Lemma $3.2([10])$. Let $f: S \rightarrow S^{3}$ be a non-conformal harmonic map and $f^{\varepsilon}$ its $\varepsilon$-transform. Then the functions $\phi, \mu$ and $\phi^{\varepsilon}, \mu^{\varepsilon}$ are related by

$$
\begin{aligned}
4 \sinh ^{2} \phi^{\varepsilon} & =\left|\mu-2 \varepsilon i \phi_{z}\right|^{2} \operatorname{sech}^{2} \phi \\
\tanh \phi^{\varepsilon}\left(\mu^{\varepsilon}+2 \varepsilon i \phi_{z}^{\varepsilon}\right) & =\tanh \phi\left(\mu-2 \varepsilon i \phi_{z}\right) .
\end{aligned}
$$

These expressions can be regarded as Bäcklund transformations for the system of integrability conditions, in the sense that from one solution $\phi, \mu$ of the second order equations (6) one can distill another solution $\phi^{\varepsilon}, \mu^{\varepsilon}$ of (6) using the first order equations (9)-(10).

In one of the main theorems of [10] we determined all non-conformal harmonic maps such that $f$ and $f^{\varepsilon}$ are congruent.

Theorem 3.3 ([10]). Let $f$ be a non-conformal harmonic map and $f^{\varepsilon}$ its $\varepsilon$ transform. Then the following statements are equivalent.

(a) The maps $f$ and $f^{\varepsilon}$ are congruent;

(b) the maps $f$ and $f^{\varepsilon}$ are $S O(4)$-congruent;

(c) $\phi=\phi^{\varepsilon}$ and $\mu=\mu^{\varepsilon}$;

(d) $\mu=\mu^{\varepsilon} \neq 0$;

(e) the function $\phi$ is constant; and

$(f)$ the function $\mu$ is constant and non-zero.

If one of these statements holds, all maps in the sequence associated to $f$ are $S O(4)$ congruent to each other and are certain reparametrisations of Clifford tori (see Theorem 4.4 in $[10]$ ). 


\section{Proof of Theorem 1.1}

Theorem 3.3 is the starting point for our discussion. Part (c) of Theorem 3.3 says that a non-conformal harmonic map $f$ is congruent to its $\varepsilon$ transform if $\phi=\phi^{\varepsilon}$ and $\mu=\mu^{\varepsilon}$. Part (d) says that it is sufficient to only assume $\mu=\mu^{\varepsilon}$. In view of these results, it is natural to ask whether $f$ and $f^{\varepsilon}$ are congruent if only $\phi=\phi^{\varepsilon}$ holds. Theorem 1.1 states that this fact is true except for one specific harmonic map.

The proof uses a result on isoparametric functions. The result states that the level sets of an isoparametric function from the plane to the real line are straight lines or circles. This fact enables us to quickly find all harmonic functions satisfying equation (15).

Before proving the theorem we give a slight extension a lemma from [10] (Lemma 4.1) and an algebraic result (Lemma 4.2).

Lemma 4.1 (Lemma 4.5 in [10]). If $\phi=\phi^{\varepsilon}$, then the following equations hold:

(a) $\mu^{\varepsilon}=\mu-4 \varepsilon i \phi_{z}$;

(b) $|\mu|=\left|\mu^{\varepsilon}\right|$;

(c) $\phi_{z \bar{z}}=2\left|\phi_{z}\right|^{2} \operatorname{csch} 2 \phi$;

(d) $|\mu|^{2}-4\left|\phi_{z}\right|^{2}=\sinh ^{2} 2 \phi$; and

(e) the function $\log \sinh \phi$ is harmonic.

Proof. The equations (a)-(d) are proven in Lemma 4.5 from [10]. To prove equation (e) note that

$$
\left(\phi_{z} \operatorname{coth} \phi\right)_{\bar{z}}=\operatorname{coth} \phi\left(\phi_{z \bar{z}}-2\left|\phi_{z}\right|^{2} \operatorname{csch} 2 \phi\right)
$$

vanishes by (c). Hence $\phi_{z} \operatorname{coth} \phi$ is holomorphic and which means that $\log \sinh \phi$ is harmonic.

Lemma 4.2. If $z_{1}, z_{2}, w$ are complex numbers satisfying $\left|z_{1}\right|=\left|z_{2}\right|$ and $\left|z_{1}-w\right|=$ $\left|z_{2}+w\right|$, then there is a real number $\lambda$ such that $z_{1}+z_{2}=i \lambda w$.

Proof. Expanding $\left|z_{1}-w\right|^{2}=\left|z_{2}+w\right|^{2}$ gives

$$
\left|z_{1}\right|^{2}-w \overline{z_{1}}-\bar{w} z_{1}+|w|^{2}=\left|z_{2}\right|^{2}+w \overline{z_{2}}+\bar{w} z_{2}+|w|^{2}
$$

Since $\left|z_{1}\right|=\left|z_{2}\right|$ this becomes $\operatorname{Re} \bar{w}\left(z_{1}+z_{2}\right)=0$ which means that $z_{1}+z_{2}$ is perpendicular to $w$. This proves the lemma.

Proof of Theorem 1.1. Assume that $\phi=\phi^{\varepsilon}$ but that $f$ and $f^{\varepsilon}$ are not congruent.

Step 1. Proof that $\mu=2 \varepsilon\left(c \operatorname{coth}^{2} \phi+i\right) \phi_{z}$ with $c \in \mathbb{R}_{0}$. From Lemma 4.1 we know that $|\mu|=\left|\mu^{\varepsilon}\right|$ and $\left|\mu-2 \varepsilon i \phi_{z}\right|=\left|\mu^{\varepsilon}+2 \varepsilon i \phi_{z}\right|$. By Lemma 4.2 there is a real valued function $\lambda$ such that $\mu+\mu^{\varepsilon}=-2 \lambda i\left(2 \varepsilon i \phi_{z}\right)=4 \lambda \varepsilon \phi_{z}$. The function $\lambda$ is non-zero because otherwise $\mu=-\mu^{\varepsilon}$ and that is impossible by Proposition 2.1. Since $\mu^{\varepsilon}=\mu-4 \varepsilon i \phi_{z}$, we get

$$
\mu=2 \varepsilon(\lambda+i) \phi_{z}
$$

Deriving $\mu$ with respect to $\bar{z}$ and using equation (c) from Lemma 4.1 gives

$$
\mu_{\bar{z}}=2 \varepsilon \lambda_{\bar{z}} \phi_{z}+2 \varepsilon(\lambda+i) \phi_{z \bar{z}}=2 \varepsilon \lambda_{\bar{z}} \phi_{z}+4 \varepsilon(\lambda+i)\left|\phi_{z}\right|^{2} \operatorname{csch} 2 \phi .
$$

On the other hand, equations (6b) and (11) give

$$
\mu_{\bar{z}}=-4 \varepsilon(\lambda-i)\left|\phi_{z}\right|^{2} \operatorname{csch} 2 \phi .
$$

Comparing the latter two equations gives

$$
\lambda_{\bar{z}} \phi_{z}=-4 \lambda\left|\phi_{z}\right|^{2} \operatorname{csch} 2 \phi .
$$

Since we have assumed that $f$ and $f^{\varepsilon}$ are not congruent the function $\phi$ is not constant by Theorem 3.3 (a) and (e). Therefore we can divide both sides by $\phi_{z}$ and 
the equation becomes $(\log |\lambda|)_{\bar{z}}=-2(\log |\tanh \phi|)_{\bar{z}}$. The solution is $\lambda=c \operatorname{coth}^{2} \phi$, $c \in \mathbb{R}_{0}$.

Step 2. The function $k=\log \sinh \phi$ is isoparametric. From Step 1 we know that

$$
|\mu|^{2}=4\left(c^{2} \operatorname{coth}^{4} \phi+1\right)\left|\phi_{z}\right|^{2} \text {. }
$$

Substituting expression (12) in equation (d) from Lemma 4.1 one finds that

$$
4 c^{2} \operatorname{coth}^{4} \phi\left|\phi_{z}\right|^{2}=\sinh ^{2} 2 \phi .
$$

The function $\phi$ and its derivative $\phi_{z}$ can be written completely in terms of $k$ :

$$
\phi=\operatorname{arcsinh}\left(e^{k}\right), \quad \phi_{z}=\frac{e^{k} k_{z}}{\sqrt{1+e^{2 k}}} .
$$

The expressions (14) simplify equation (13) to

$$
\left|k_{z}\right|=\frac{e^{2 k}}{|c|} .
$$

From Lemma 4.1 (e) we also know that $k$ is a harmonic function. Therefore the length of the gradient and the laplacian of $k$ are constant along each level curve, which means exactly that the function $k$ is isoparametric.

Step 3. Obtaining the invariants. The gradient of $k$ vanishes nowhere by (15) so the level curves of $k$ are smooth. A result on isoparametric functions (see [2] or $[14$, p. 116]) states that the level curves of $k$ are either parallel lines or concentric circles.

If the level curves are lines, $k$ depends on only one cartesian coordinate. The function $k$ is harmonic and depends on one coordinate, hence it must be a first degree polynomial function. However, such a map cannot be a solution of (15), so this case cannot occur.

If the level curves are concentric circles, then after a suitable translation $z \mapsto$ $z+z_{0}$ of the complex coordinate, the function $k$ only depends on the radial coordinate $r=\sqrt{x^{2}+y^{2}}$. Since $k$ is harmonic, $k=a \log r+b$, with $a, b \in \mathbb{R}$. Substituting $k$ in (15) gives $|a| c=e^{2 b} r^{2 a+1}$. So $a$ must be equal to $-1 / 2$ and thus $b=1 / 2 \log (|c| / 4)$. Hence

$$
k=\frac{1}{2} \log \frac{c}{2 r}
$$

are the harmonic functions that satisfy (15). The expressions for $\phi$ and $\mu$ can be calculated from $\phi=\operatorname{arcsinh}\left(e^{k}\right)$ and $\mu=2 \varepsilon\left(c \operatorname{coth}^{2} \phi+i\right) \phi_{z}$. If one reparametrises the harmonic function by performing the dilation $z \mapsto|c| z / 4$, one obtains the expressions (1) for $\phi$ and $\mu$ in the statement

\section{The Bonnet PROBlem FOR NON-CONFORMAL HARMONIC MAPS}

Consider a Riemann surface $S$ and two harmonic maps $f_{1}, f_{2}: S \rightarrow S^{3}$ from $S$ into the round 3 -sphere $S^{3}$. The holomorphic quadratic differentials $\Theta_{k}, k=1,2$, are defined by

$$
\Theta_{k}=\left\langle\left(f_{k}\right)_{z},\left(f_{k}\right)_{z}\right\rangle d z^{2}
$$

where $z$ is an arbitrary complex coordinate on $S$. Assume that $\Theta_{1}=\Theta_{2}$. Two cases can occur.

(a) If $\Theta_{1}=\Theta_{2}$ is identically zero, the harmonic maps $f_{k}, k=1,2$, are conformal and thus they are minimal isometric immersions of $S$ into the three-sphere $S^{3}$.

(b) If $\Theta_{1}=\Theta_{2}$ is not identically zero, the set of zeros consists of isolated points. Now we state the Bonnet problem for non-conformal harmonic maps.

Bonnet problem. Consider two regular non-conformal harmonic maps $f_{1}, f_{2}: S \rightarrow$ $S^{3}$. Assume that $\Theta_{1}=\Theta_{2}$ and $\left|\left(f_{1}\right)_{z}\right|=\left|\left(f_{2}\right)_{z}\right|$. Are the two maps necessarily congruent? 
The problem presented here is a non-conformal analogue of the classical Bonnet problem. Let $X_{1}$ and $X_{2}$ be two isometric immersions of a Riemann surface $S$ into $\mathbb{R}^{3}$ for which the induced metrics and mean curvature functions agree. The classical Bonnet problem then asks whether the two immersions $X_{1}$ and $X_{2}$ are congruent. Bonnet indicated that that there are three types of surfaces who have an isometric but non-congruent 'sister surface'. One of these types of surfaces are constant mean curvature (CMC) surfaces.

The Bonnet problem for harmonic maps looks basically the same as the one for CMC surface, but there is an essential difference. First, we have to assume that $\Theta_{1}=\Theta_{2}$ for the problem to be well-stated. It means that the conformal structure induced on $S$ is the same for the maps $f_{1}$ and $f_{2}$. This assumption allows us to choose a complex coordinate adapted to both $f_{1}$ and $f_{2}$ (see equation (16)). Note that if $f_{1}$ and $f_{2}$ are isometric immersions, then both holomorphic quadratic differentials are automatically equal to zero.

Secondly we assume that $\left\langle\left(f_{1}\right)_{z},\left(f_{1}\right)_{\bar{z}}\right\rangle=\left\langle\left(f_{2}\right)_{z},\left(f_{2}\right)_{\bar{z}}\right\rangle$. Choosing an adapted coordinate $z$, this means that $\phi_{1}=\phi_{2}$ (see (17) below). This assumption is analogous to assuming that the metrics agree in the classical Bonnet problem.

Finally, there is a correspondence between harmonic maps to $S^{3}$ and $H$-surfaces $X$ in $\mathbb{R}^{3}$, i.e. immersions of a surface that satisfy the equation $X_{x x}+X_{y y}=2 X_{x} \times X_{y}$ (see Proposition 5.2 in [10]). If the harmonic map to $S^{3}$ is a minimal surface then the corresponding $H$-surface is CMC. In this case the correspondence is the Lawson correspondence $([13])$.

However, the Bonnet problems for CMC surfaces and harmonic maps are not entirely the same. The Gauss-Codazzi equations of a CMC surface are invariant under the transformation $Q \rightarrow e^{i t} Q, t \in \mathbb{R}$, where $Q d z^{2}$ is the Hopf differential of the CMC surface. Applying the Bonnet theorem then gives a 1-parameter family of isometric surfaces that all have the same constant mean curvature.

To a non-conformal harmonic map one can also associate a 1-parameter family of harmonic maps by transforming the invariants of the map. However, the invariant $\phi$ of the map does not remain invariant under this transformation (see Lemma 5.1). Therefore the Bonnet problem for non-conformal harmonic maps is not trivial.

Lemma 5.1. Consider a non-conformal harmonic map $f: S \rightarrow S^{3}$. The integrability conditions (6) are invariant under the transformation

$$
\phi(z) \rightarrow \phi\left(e^{i t} z\right), \quad \mu(z) \rightarrow e^{i t} \mu\left(e^{i t} z\right), \quad t \in \mathbb{R}
$$

and thus one obtains a 1-parameter family $f_{t}: S \rightarrow S^{3}, t \in \mathbb{R}$ of non-conformal harmonic maps.

Proof. By Proposition 2.1 we know that each pair of functions $\phi$ and $\mu$ that satisfies the system of equations (6) gives a non-conformal harmonic map. We show that if $\phi$ and $\mu$ are solutions of (6) then

$$
\begin{aligned}
& \hat{\phi}(z)=\phi\left(e^{i t} z\right), \\
& \hat{\mu}(z)=e^{i t} \mu\left(e^{i t} z\right)
\end{aligned}
$$

for every $t \in \mathbb{R}$ are also solutions of this system.

Note that $\hat{\phi}_{z}(z)=e^{i t} \phi_{z}\left(e^{i t} z\right)$ and $\hat{\phi}_{z \bar{z}}(z)=\phi_{z \bar{z}}\left(e^{i t} z\right)$ and therefore $\hat{\phi}$ and $\hat{\mu}$ satisfy (6a). Also note that $\hat{\mu}_{\bar{z}}(z)=\mu_{\bar{z}}\left(e^{i t} z\right)$ and hence $\hat{\phi}$ and $\hat{\mu}$ also satisfy (6b).

We now present the first results for the Bonnet problem of non-conformal harmonic maps. Consider two regular non-conformal harmonic maps $f_{1}$ and $f_{2}$ from a Riemann surface $S$ into $S^{3}$. We always assume that $\Theta_{1}=\Theta_{2}$ and $\left|\left(f_{1}\right)_{z}\right|=\left|\left(f_{2}\right)_{z}\right|$. 
On the open subset where $\Theta_{k}$ does not vanish, one can choose a complex coordinate $z$ such that

$$
\left\langle\left(f_{1}\right)_{z},\left(f_{1}\right)_{z}\right\rangle=\left\langle\left(f_{2}\right)_{z},\left(f_{2}\right)_{z}\right\rangle=-1,
$$

which means that $z$ is an adapted coordinate for both $f_{1}$ and $f_{2}$.

By (3) there exist Im $\mathbb{H}$-valued functions $\alpha_{k}$ and $\beta_{k}, k=1,2$, such that $\left(f_{k}\right)_{z}=$ $\frac{1}{2} f\left(\alpha_{k}-i \beta_{k}\right)$ and by equation (16) the functions $\alpha_{k}$ and $\beta_{k}$ satisfy

$$
\left|\alpha_{k}\right|=2 \sinh \phi_{k}, \quad\left|\beta_{k}\right|=2 \cosh \phi_{k}
$$

for some real functions $\phi_{k}, k=1,2$. Since the maps $f_{k}$ are regular, $f_{x}$ and $f_{y}$ are linearly independent so the functions $\phi_{k}$ are positive. Since $\left|\left(f_{k}\right)_{z}\right|^{2}=\cosh 2 \phi_{k}$, $k=1,2$, we have

$$
\left|\left(f_{1}\right)_{z}\right|=\left|\left(f_{2}\right)_{z}\right| \quad \Leftrightarrow \quad \phi_{1}=\phi_{2}
$$

Lemma 5.2. Consider two regular harmonic map $f_{1}, f_{2}: S \rightarrow S^{3}$ satisfying the assumptions of the Bonnet problem. Then the following statements are equivalent.

(a) $\left\langle\left(f_{1}\right)_{z},\left(f_{2}\right)_{z}\right\rangle d z^{2}$ is a holomorphic differential.

(c) The angle between $f_{1}$ and $f_{2}$ is constant at each point on $S$.

Proof. The proof follows from the following calculation:

$$
\begin{aligned}
\left\langle\left(f_{1}\right)_{z},\left(f_{2}\right)_{z}\right\rangle_{\bar{z}} & =\left\langle\left(f_{1}\right)_{z \bar{z}},\left(f_{2}\right)_{z}\right\rangle+\left\langle\left(f_{1}\right)_{z},\left(f_{Z}\right)_{z \bar{z}}\right\rangle \\
& =-\left|\left(f_{1}\right)_{z}\right|^{2}\left\langle f_{1},\left(f_{2}\right)_{z}\right\rangle-\left|\left(f_{2}\right)_{z}\right|^{2}\left\langle\left(f_{1}\right)_{z}, f_{2}\right\rangle \\
. & =-\left|\left(f_{1}\right)_{z}\right|^{2}\left\langle f_{1}, f_{2}\right\rangle_{z}
\end{aligned}
$$

where we used in the last step that $\left|\left(f_{1}\right)_{z}\right|=\left|\left(f_{2}\right)_{z}\right|$.

Corollary 5.3. Let $f$ be a non-conformal harmonic map $f$ with adapted coordinate $z$ and let $f^{\varepsilon}$ be its $\varepsilon$-transform. Then $\phi=\phi^{\varepsilon}$ if and only if $\left\langle f_{z}, f_{z}^{\varepsilon}\right\rangle d z^{2}$ is a holomorphic differential.

Proof. If $\phi=\phi^{\varepsilon}$ then by (17) $\left|f_{z}\right|=\left|f_{z}^{\varepsilon}\right|$. By Theorem 3.1 the coordinate $z$ is also an adapted coordinate for $f^{\varepsilon}$. Therefore the conditions of the Bonnet problem are satisfied. By definition of the transform $\left\langle f, f^{\varepsilon}\right\rangle=0$. Hence by Lemma 5.2 $\left\langle\left(f_{1}\right)_{z},\left(f_{2}\right)_{z}\right\rangle d z^{2}$ is holomorphic. This proves the first implication.

Conversely assume that $\left\langle\left(f_{1}\right)_{z},\left(f_{2}\right)_{z}\right\rangle$ is holomorphic. In Lemma 3.3 in [10] we showed that $\left\langle f_{z}, f_{z}^{\varepsilon}\right\rangle=\tanh \phi\left(\mu-2 \varepsilon i \phi_{z}\right)$ A calculation using (6) and (9) shows that

$$
0=\left(\tanh \phi\left(\mu-2 \varepsilon i \phi_{z}\right)\right)_{\bar{z}}=2 \varepsilon i\left(\sinh ^{2} \phi-\sinh ^{2} \phi^{\varepsilon}\right),
$$

so $\phi=\phi^{\varepsilon}$.

Lemma 5.4. Let $f_{1}$ and $f_{2}$ be regular non-conformal harmonic maps that satisfy the assumptions of the Bonnet problem and let $\phi_{1}, \mu_{1}$ respectively $\phi_{2}, \mu_{2}$ be their invariants. Then $\left|\mu_{1}\right|=\left|\mu_{2}\right|$. Moreover $\left(\mu_{1}^{2}-\mu_{2}^{2}\right) d z^{4}$ is a holomorphic quartic differential.

Proof. The equation $\left|\mu_{1}\right|=\left|\mu_{2}\right|$ follows from (6a) and (17). It can be shown by a standard calculation that

$$
\left(\mu_{1}^{2}-\mu_{2}^{2}\right) d z^{4}=\left(\left\langle\left(f_{1}\right)_{z z}, N_{1}\right\rangle^{2}-\left\langle\left(f_{2}\right)_{z z}, N_{2}\right\rangle^{2}\right) d z^{4}
$$

does not depend on the choice of the complex coordinate $z$. Furthermore by $(6 \mathrm{~b})$

$$
\left(\mu_{1}^{2}-\mu_{2}^{2}\right)_{\bar{z}}=4\left(\left|\mu_{2}\right|^{2}-\left|\mu_{1}\right|^{2}\right) \phi_{z} \operatorname{csch} 2 \phi=0,
$$

with $\phi=\phi_{1}=\phi_{2}$ so the quartic differential is holomorphic.

Corollary 5.5. If $f_{1}$ and $f_{2}$ are not congruent, then the set $\left\{p \in S \mid \mu_{1}(p)=\right.$ $\left.\pm \mu_{2}(p)\right\}$ consists of isolated points. 
Proof. If $\mu_{1}^{2}=\mu_{2}^{2}$, then $\mu_{1}= \pm \mu_{2}$ so $f_{1}$ and $f_{2}$ are congruent by a rotation resp. reflection by Proposition 2.1. If $\mu_{1}^{2} \neq \mu_{2}^{2}$, then the zeros of the quartic differential are isolated, since it is a holomorphic differential.

\section{REFERENCES}

1. M. Antic and L. Vrancken, Sequences of minimal surfaces in $S^{2 n+1}$, Israel J. Math. 179 (2010), no. 1, 493-508.

2. J. Berndt, C.E. Olmos, and S. Console, Submanifolds and holonomy, Monographs and Research Notes in Mathematics, CRC Press, 2003.

3. A.I. Bobenko and U. Eitner, Painleve equations in the differential geometry of surfaces, Lecture Notes in Mathematics, no. nr. 1753, SFB 288, 2000

4. J. Bolton and L. Vrancken, Transforms for minimal surfaces in the 5-sphere, Differential Geom. Appl. 27 (2009), 34-46.

5. O. Bonnet, Mémoire sur la théorie des surfaces applicables., J. Ec. Polyt. 42 (1867), 72-92.

6. E. Cartan, Sur les couples de surfaces applicables avec conservation des courbures principales, Bull. Sci. Math. II 66 (1942), 55-72 and 74-85.

7. W.H. Chen and H.Z. Li, Bonnet surfaces and isothermic surfaces, Result Math. 31 (1997), 40-52.

8. X. Chen and C.K. Peng, Lecture Notes in Math., vol. 1369, ch. Deformations of surfaces preserving principal curvatures, pp. 63-70, Springer Verlag, 1989.

9. S.S. Chern, Differential Geometry and Complex Analysis, Deformation of surfaces preserving principal curvatures (I. Chavel and H.M. Farkas, eds.), Springer, New York, 1985, pp. 155-163.

10. B. Dioos, J. Van der Veken, and L. Vrancken, Sequences of harmonic maps in the 3-sphere, Math. Nachr. 17-18 (2015), 1917-2152.

11. J. Gálvez, A. Martínez, and P. Mira, The Bonnet problem for surfaces in homogeneous 3manifolds, Commun. Anal. Geom. 16 (2008), no. 5, 907-935.

12. H. He, H. Ma, and E. Wang, Lagrangian Bonnet pairs in complex space forms, arXiv:1503.08566, 2015.

13. H.B. Lawson, Complete minimal surfaces in $S^{3}$, Ann. Math. 92 (1970), 335-374.

14. P. Tondeur, Geometry of foliations, Monographs in Mathematics, Springer, 1997.

Bart Dioos, Joeri Van der Veken, Ku Leuven, Departement Wiskunde, CelestijnenLAAN 200B - Bus 2400, 3001 LEuven, Belgium

E-mail address: bart.dioos@kuleuven.be

E-mail address: joeri.vanderveken@kuleuven.be 